06

\title{
Индуцированная электрическим полем динамика структурного упорядочения в тройных системах $\mathrm{Pbln}_{1 / 2} \mathrm{Nb}_{1 / 2} \mathrm{O}_{3}-\mathrm{PbMg}_{1 / 3} \mathrm{Nb}_{2 / 3} \mathrm{O}_{3}-x \mathrm{PbTiO}_{3}$
}

\author{
(С) Л.С. Камзина, Л.А. Кулакова \\ Физико-технический институт им. А.Ф. Иоффре РАН, \\ Санкт-Петербург, Россия \\ E-mail: kamzin@mail.ioffe.ru
}

(Поступила в Редакцию 12 июля 2016 г.)

\begin{abstract}
Исследована индуцированная электрическим полем динамика фазового перехода в сегнетоэлектрическую фазу в кристаллах $\mathrm{PbIn}_{1 / 2} \mathrm{Nb}_{1 / 2} \mathrm{O}_{3}-\mathrm{PbMg}_{1 / 3} \mathrm{Nb}_{2 / 3} \mathrm{O}_{3}-x \mathrm{PbTiO}_{3}(x=25$ и $32 \%$ ) в различных геометриях приложения внешнего электрического поля $E\|[001], E\|[011]$. При комнатной температуре проведены одновременные измерения оптического пропускания, скорости и затухания звука. Обнаружено, что изменение упругих свойств наступает позже, чем изменение оптического пропускания, что связано с разными размерами полярных областей и времен релаксации, на которые реагируют свет и звук. Установлено, что в интервале полей $0-2 \mathrm{kV} / \mathrm{cm}$ при $E \|[001]$ в обоих кристаллах в результате фазового перехода происходит только частичная поляризация образца, в то время как в кристалле РТ32 при $E \|[011]$ в том же интервале полей фазовый переход завершается практически полной поляризацией образца.
\end{abstract}

DOI: 10.21883/FTT.2017.02.44050.293

\section{1. Введение}

Релаксорные сегнетоэлектрики - это большой класс соединений, которые вызывают повышенный интерес исследователей благодаря своим необычным диэлектрическим и электромеханическим свойствам. Основной чертой релаксоров является сильная частотная дисперсия диэлектрической проницаемости, отсутствие макроскопической спонтанной поляризации при охлаждении в нулевом поле и возникновение сегнетоэлектрического поведения только после охлаждения в электрическом поле. Классический релаксор магнониобат свинца $\mathrm{PbMg}_{1 / 3} \mathrm{Nb}_{2 / 3} \mathrm{O}_{3}$ (PMN) обнаруживает в среднем кубическую симметрию ниже максимума диэлектрической проницаемости вплоть до очень низких температур. Необычные свойства этих материалов связаны с появлением локальных пространственно ориентированных сегнетоэлектрических наноразмерных областей (полярные нанообласти PNR) [1,2]. Эти динамические области, внедренные в разупорядоченную кубическую матрицу, характеризуются локальной ромбоэдрической симметрией. Они возникают при температуре Бернса $\left(T_{B} \sim 620-650 \mathrm{~K}\right)$ и имеют размеры $2-3 \mathrm{~nm}$. С понижением температуры размеры их возрастают и достигают $\sim 10-30 \mathrm{~nm}$ ниже температуры замерзания $T_{f}$. При этой температуре возникает стеклоподобное состояние c замороженной локальной поляризацией. Кроме того, в релаксорах наблюдаются статические нано- и микродоменные структуры [3-5]. Таким образом, релаксорные сегнетоэлектрики демонстрируют структурную иерархию и обнаруживают высокоупорядоченную структуру только на мезоскопическом масштабе.
Общей чертой всех релаксоров, таких как $\mathrm{PMN}, \quad \mathrm{PbMg}_{1 / 3} \mathrm{Nb}_{2 / 3} \mathrm{O}_{3}-x \mathrm{PbTiO}_{3}, \quad(\mathrm{PMN}-x \mathrm{PT})$, $\mathrm{PbIn}_{1 / 2} \mathrm{Nb}_{1 / 2} \mathrm{O}_{3}-\mathrm{PbMg}_{1 / 3} \mathrm{Nb}_{2 / 3} \mathrm{O}_{3}-x \mathrm{PbTiO}_{3} \quad$ (PIN $-\mathrm{PMN}-x \mathrm{PT})$ и ряда других, является случайное расположение ионов в кристаллографически эквивалентных положениях. Случайно ориентированные локальные электрические и упругие поля служат причиной возникновения PNR, которые являются предшественниками сегнетоэлектрического фазового перехода. Эволюция полярных нанообластей связана в релаксорах с четырьмя характерными температурами: 1 - температура Бернса $T_{B} \sim 650 \mathrm{~K}$, при которой появляются динамические PNR, 2 - промежуточная температура $T_{i m}$, ниже которой динамические PNR трансформируются в статические с быстрым ростом размеров, 3 - частотно-зависимая температура максимума диэлектрической проницаемости $T_{m}$ и 4 - температура сегнетоэлектрического фазового перехода $T_{C}$ (или температура замерзания $T_{f}$ в случае, когда этот переход отсутствует, как в PMN). Различные исследования релаксорных сегнетоэлектриков были предприняты, чтобы понять их необыкновенные свойства. Так, например, появление динамических PNR послужило причиной отклонения показателя преломления и скорости звука от обычной линейной температурной зависимости [6,7]. Статические PNR меняют поведение теплового расширения [8]. В замороженном релаксорном состоянии PNR приобретают слабую кинетику, но не образуют дальнего порядка. PNR ведут себя как большие дипольные моменты, совершающие тепловые флуктуации между эквивалентными потенциальными ямами, и наблюдаемые свойства релаксоров связаны с переориентацией этих диполей. Широкий спектр времен релаксации спонтанной поля- 
ризации связан как с распределением величин потенциальных барьеров, разделяющих состояния с различной ориентацией, так и с разными размерами полярных областей. Это является основной чертой неравновесного состояния стекольной системы. Свойства кристалла в таком неравновесном состоянии должны зависеть от времени. Это было подтверждено рядом авторов при изучении диэлектрических свойств [9-11] и нашими работами по оптическим и акустическим исследованиям $[12,13]$. В нашей работе [13] мы изучали кинетику прохождения сегнетоэлектрического фазового перехода в составах PIN-PMN- $x$ PT, расположенных на разном расстоянии от морфотропной фазовой границы (МФГ), используя оптические и акустические измерения.

Мы показали, что во всех исследованных кристаллах PIN-PMN-xPT $(x=23,25,32 \%)$ индуцированный фазовый переход в сегнетоэлектрическую фазу при приложении электрического поля, превосходящего пороговое поле, при температурах ниже $T_{C}$ зависит от времени и проходит в два этапа: первый этап инкубационный период, связанный с незначительным изменением поляризации в части образца, которая находится в стеклоподобной фазе. В течение этого периода наблюдается практически логарифмическая зависимость оптических и акустических характеристик. Второй этап происходит спустя время $t_{d}$ (время задержки) и связан с увеличением размера полярных областей, быстрым ростом поляризации и образованием дальнего порядка.

Мы обнаружили, что время задержки $t_{d}$ сильно зависит не только от величины приложенного поля, но и от времени, прошедшего между отжигом образца и временем начала измерения. При этом было замечено, что времена задержки $t_{d}$, полученные из оптических и акустических измерений при одних и тех же условиях измерения, отличаются друг от друга. Возникает вопрос, в чем причина таких различий. Мы предположили, что причина этого различия связана с разными размерами полярных областей и соответственно времен релаксаций, определяющих рассеяние света и изменение упругих свойств решетки. Однако эти наблюдения носили только качественный характер, так как измерения проводились на разных установках, что существенно затрудняло точное воспроизведение условий измерений на оптической и акустической установках.

В данной работе будет исследована индуцированная электрическим полем динамика фазового перехода в сегнетоэлектрическую фазу в кристаллах PIN-PMN $-x$ PT $(x=25$ и $32 \%)$ и ее связь с различным содержанием РТ. Будут проведены одновременные измерения изменений оптического пропускания и упругих свойств, как в разных электрических полях, так и при разных временах между отжигом образцов и началом измерений. Состав с малой концентрацией РТ $(x=25 \%)$ расположен значительно ниже МФГ и является, практически, релаксором. В кристаллах с большей концентрацией РТ $(x=32 \%)$, которые находятся вблизи МФГ $\left(T_{r t} \sim 385 \mathrm{~K}\right)$, доля релаксорной фазы невелика.

\section{2. Методика эксперимента}

Кристаллы выращивались прямо из расплава модифицированным методом Бриджмена [14]. Были получены высококачественные монокристаллы 20PIN-55PMN-25PT (PT25) и 33PIN-35PMN-32PT) (РТ32). Кристаллы ориентировались и затем разрезались вдоль плоскостей (001) и (011). Образцы с размерами $3 \times 3 \times 4 \mathrm{~mm}$ разной ориентации вырезались из одного куска кристалла, чтобы минимизировать изменения состава, возникающего из-за сегрегации во время роста. Грани образцов подвергались оптической полировке. Перед каждым измерением образцы отжигались в течение часа при температуре $\sim 500 \mathrm{~K}$. После отжига образцы охлаждались без поля до комнатной температуры, при которой к ним прикладывалось постоянное электрическое поле с напряженностью, достаточной для индуцирования фазового перехода в сегнетоэлектрическое состояние. Чтобы избежать пробоя кристалла при приложении больших электрических полей, в данной работе использовались небольшие электрические поля, которые приводят к частичной поляризации образца. Поскольку результаты, полученные при одном и том же поле, были вполне воспроизводимы, то можно было получать различную степень поляризации путем изменения величины поля.

Измерения проводились при комнатной температуре. Электрическое поле прикладывалось в направлении [001] для кристалла РТ25, и в направлениях [001] и [011] для кристалла РТ32. После приложения поля регистрировались изменения оптического пропускания, затухания и скорости звука со временем.

Пропускание света измерялось в перпендикулярном [100] направлении. Для оптических измерений использовался $\mathrm{He}-\mathrm{Ne}$-лазер $(\lambda=632.8 \mathrm{~nm})$. Оптический сигнал регистрировался быстродействующим фотоприемником (ФЭУ28). Импульс света формировался дисковым прерывателем. Им же вырабатывался импульс синхронизации запуска ВЧ генератора, вырабатывающего акустический импульс. Для измерения акустических свойств кристаллов использовалась эхо-импульсная методика. Ультразвуковые импульсы частотой $20 \mathrm{MHz}$ и длительностью до $0.5 \mu \mathrm{s}$ возбуждались (с помощью генератора сигналов AFG фирмы INSTEK) резонансными пластинками из пьезоэлектрической керамики, приклеиваемыми к образцу. Широкополосный (полоса $200 \mathrm{MHz}$ ) осциллограф (фирмы Tectronix) и камера Sony Cyber-shot использовались для фиксированной и непрерывной записи поведения эхо-импульсов. Точность измерения изменения скорости звука не хуже $10^{-3}$. При акустических измерениях электрическое поле совпадало по направлению с распространением звуковой волны.

\section{3. Экспериментальные результаты и их обсуждение}

3.1. Электрическое поле в направлен и и [001]. На рис. 1, $a, b$ в качестве примера приведены 
изменения оптического пропускания и скорости звука со временем в электрическом поле для кристаллов РТ25 и РТ32, полученные при одновременном измерении оптических и акустических характеристик. Как следует из рентгеновских и нейтронных исследований, симметрия образцов РТ25 при комнатной температуре в отсутствие электрического поля в целом кубическая с внедрением неупорядоченных хаотически расположенных областей ромбоэдрической фазы $\left(T_{f} \sim 376 \mathrm{~K}\right)$, а образцов РТ32 ромбоэдрическая с отдельными областями моноклинной фазы и небольшой долей релаксорной стеклоподобной фазы (температура морфотропного фазового перехода $\left.T_{r t} \sim 385 \mathrm{~K}\right)$ [15].

Как мы уже сообщали ранее в работах $[13,14]$ и как видно из рис. 1, фазовый переход в сегнетоэлектрическое состояние проходит в два этапа. Во время первого этапа кинетика в основном определяется приблизительно логарифмическим ростом поляризации в стекольной фазе, при этом в кристалле РТ25 формируются малые области ромбоэдрической фазы. Наличие такой логарифмической зависимости для кристаллов РТ32 служит подтверждением частичного присутствия стекольной фазы и в этом кристалле. В кристалле РТ25 после перехода индуцируется ромбоэдрическая фаза, а в кристалле РТ32 моноклинная [15].
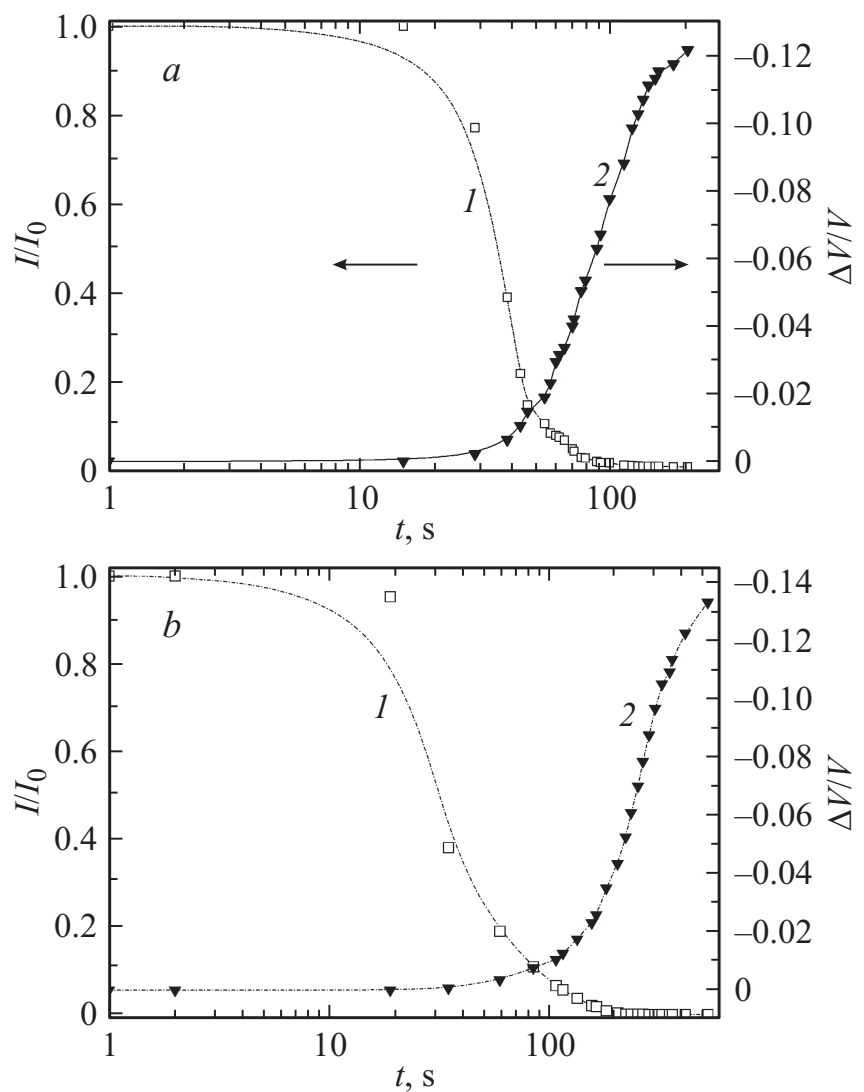

Рис. 1. Изменения оптического пропускания $I / I_{0}$ (1) и скорости звука $(\Delta V / V)(2)$ со временем в электрическом поле $(E, \mathrm{kV} / \mathrm{cm})$ в кристаллах РТ25 (a) и РТ32 $(b): a-E=1.5$, $b-E=1.97$.
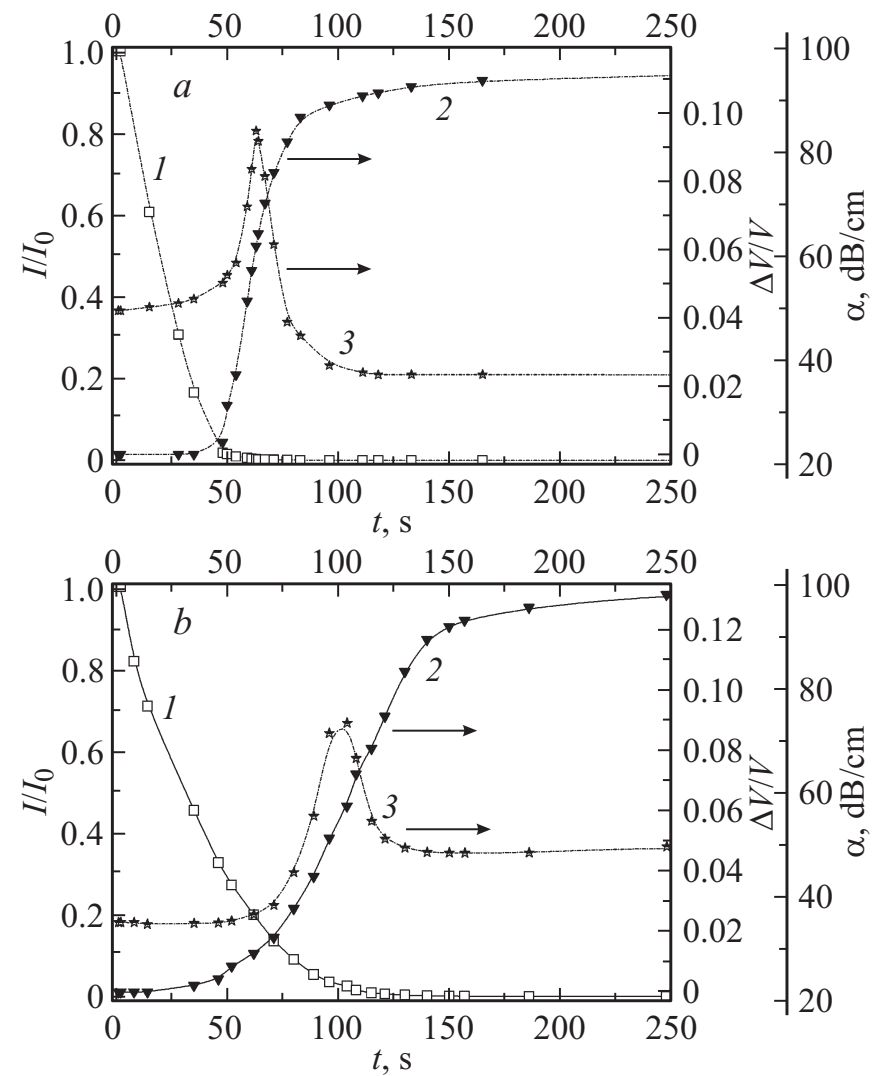

Рис. 2. Динамика изменений оптического пропускания (1), скорости звука (2) и поглощения звука $\alpha(3)$, происходящих при фазовом переходе в электрическом поле $E=1.6 \mathrm{kV} / \mathrm{cm}$ в кристалле РТ25, при различных временах $t_{r}$, прошедших между отжигом кристаллов и началом измерений: $a-1 \mathrm{~h} 45 \mathrm{~s}$, $b-4 \mathrm{~h}$.

На рис. 2, $a, b$ представлена динамика изменения оптического пропускания и акустических свойств (скорость звука и затухание) для кристаллов РТ25 в электрическом поле при разных временах, прошедших между отжигом образца и началом измерений $\left(t_{r}\right)$. В нашей работе [13] было показано, что измеряемые параметры сильно зависят от времени между отжигом кристалла и началом измерений. Из рис. 2 видно, что с увеличением $t_{r}$ увеличивается время прохождения фазового перехода: кривые изменения пропускания света и скорости звука становятся более пологими. Это, с одной стороны, связано с более широким спектром времен релаксации, который возникает при больших временах, прошедших между отжигом образцов и началом измерений. С течением времени увеличиваются размеры полярных областей, что приводит к увеличению времени релаксации. С другой стороны, с течением времени формируются связанные со старением релаксора внутренние поля, которые приводят к экранировке и уменьшению внешнего приложенного поля. В меньшем поле переход в фазу с дальним порядком проходит значительно медленнее. 


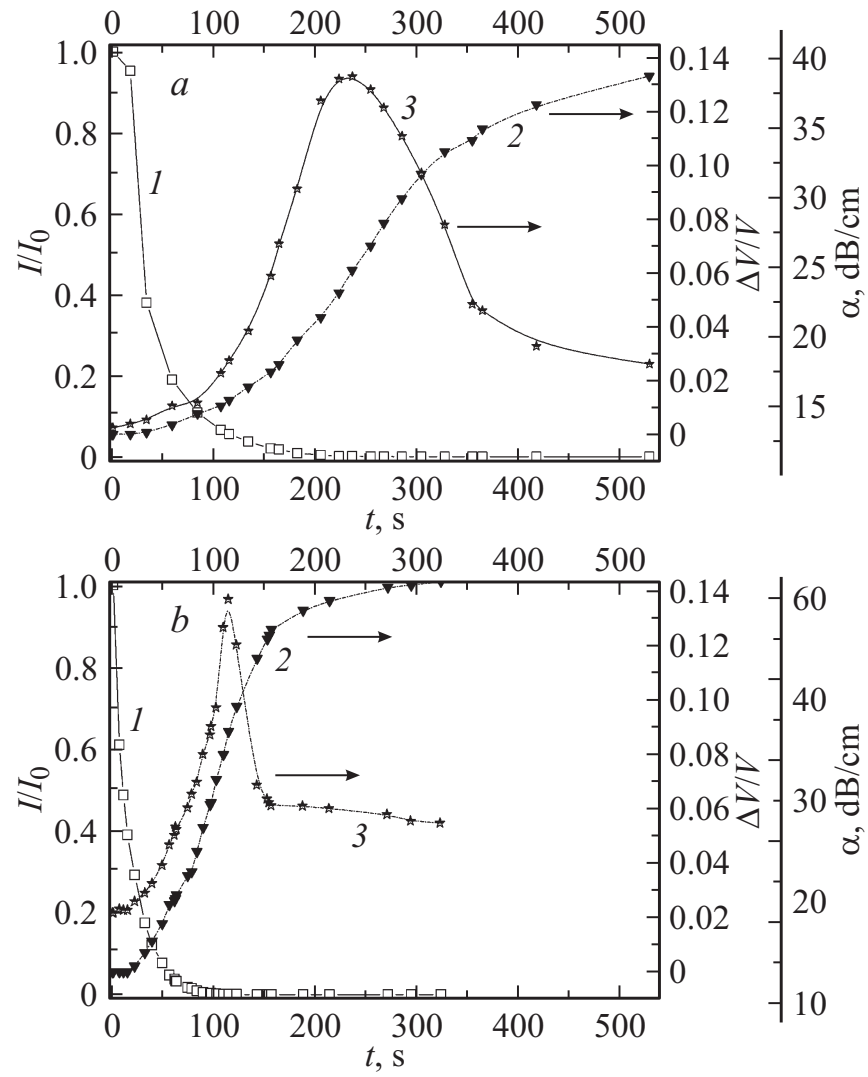

Рис. 3. Кинетика изменений оптического пропускания (1), скорости звука (2) и поглощения звука (3) в кристалле РТ32, происходящих при фазовом переходе, в разных электрических полях $E, \mathrm{kV} / \mathrm{cm}$, приложенных в направлении [001]: $a-1.97$, $b-2.1$.

На рис. 3, $a, b$ показаны изменения оптического пропускания и акустических свойств в кристаллах РТ32 в разных электрических полях, приложенных в направлении [001]. Как видно из рис. 2 и 3, фазовый переход растянут во времени: изменение упругих свойств наступает позже, чем изменение оптического пропускания. Чем же определяется изменение оптического пропускания и упругих свойств? Изменение оптических свойств, главным образом, связано с изменением размеров неоднородностей. С течением времени при приложении электрического поля число областей с локальной поляризацией возрастает. Увеличиваются и их размеры. Как только размеры становятся одного порядка или больше длины волны света $(\sim 630 \mathrm{~nm})$, происходит уменьшение оптического пропускания вследствие рассеяния света.

Соответственно изменениям размеров полярных областей изменяются и их времена релаксации. Как видно из рис. 2 и 3 (кривые 2,3), в процессе фазового перехода наблюдается резкое изменение затухания и скорости звука. Именно такое поведение может быть обусловлено релаксационными процессами. Релаксационный вклад в изменение поглощения и скорости звука связан с изменением характерных времен релаксации: $\alpha \sim \omega^{2} \tau /\left(1+\omega^{2} \tau^{2}\right), \Delta v / v \sim \omega^{2} \tau^{2} /\left(1+\omega^{2} \tau^{2}\right)$, где $\alpha-$ коэффициент поглощения, $\Delta v / v$ - относительное изменение скорости звука, $\tau$ - время релаксации спонтанной поляризации. Максимум затухания и резкое изменение скорости звука достигается при $\omega \tau \sim 1$, т.е. когда $\tau$ становится одного порядка с периодом звуковой волны (в нашем случае $\sim 50 \mathrm{~ns}$ ). Таким образом, изменение акустических свойств является информативной характеристикой, позволяющей делать выводы об изменении времен релаксации, а значит и размеров полярных областей в процессе фазового перехода.

Скорость и время прохождения фазового перехода для кристалла РТ32, также как и для кристалла РТ25, зависят от величины приложенного поля. Ширина пика затухания также зависит от величины приложенного поля. В меньшем поле (рис. 3, a) фазовый переход проходит медленнее. Из рис. 3 видно, что величина затухания в исходной фазе до перехода в кристалле РТ32 ниже, чем после перехода. Кристалл РТ32 при комнатной температуре находится в основном в ромбоэдрической фазе. При фазовом переходе в электрическом поле возникает менее симметричная моноклинная фаза, в которой затухание больше.

Поскольку акустические аномалии наблюдаются позже оптических, естественно предположить, что появляются они при возникновении более крупномасштабных полярных областей. Следует особо отметить, что изменение упругих свойств (для обоих кристаллов) в случае приложения электрического поля вдоль [001] происходит быстрее с увеличением величины внешнего поля. Однако скачкообразного изменения, как это должно было бы быть при полной поляризации образца, не наблюдалось. Это говорит о том, что при приложении небольшого электрического поля в направлении [001] в результате фазового перехода происходит только частичная поляризация образца, и дальний порядок возникает не во всем кристалле. Именно с неполной поляризацией образца мы связываем отсутствие увеличения оптического пропускания при изменении акустических свойств.

3.2. Электрическое поле в направлени и [011]. Экспериментальные результаты [16] показали, что направление [011], также как и направление [001], является еще одним направлением поляризации, в котором наблюдается большой пьезоэффект. В образцах, поляризованных в направлении [011], было обнаружено присутствие сегнетоэлектрической орторомбической фазы $(O)$, в то время как в образцах, поляризованных в направлении [001], возникала моноклинная фаза [16].

На рис. 4, $a-c$ представлены изменения оптического пропускания и акустических свойств в кристалле РТ32 в разных электрических полях, приложенных вдоль направления [011]. В отсутствие поля кристалл находится в ромбоэдрической фазе с небольшой долей областей релаксорной стеклоподобной фазы. Приложение электрического поля вдоль направления [011], согласно данным работ [17,18] и нашим исследованиям [12], 

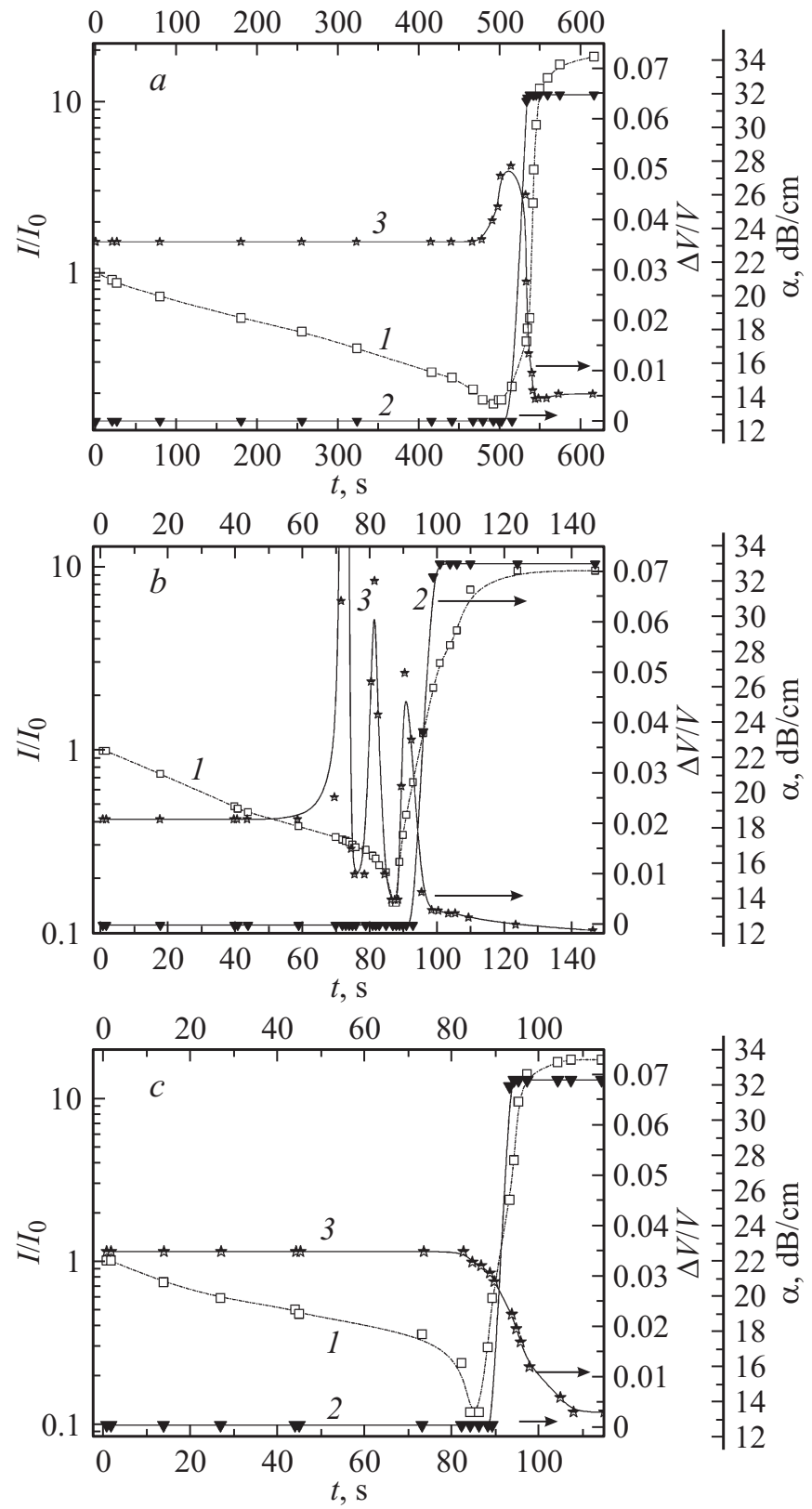

Рис. 4. Изменения оптического пропускания (1), скорости звука (2) и затухания звука (3) со временем в электрическом поле $E, \mathrm{kV} / \mathrm{cm}$, приложенном в направлении [011] в кристалле PT32: $a-E=1.59, b-E=1.69, E=1.87$.

индуцирует переход из ромбоэдрической в орторомбическую фазу. Этот фазовый переход происходит с временной задержкой, так как в образце имеются доли областей как релаксорной стеклоподобной, так и ромбоэдрической фазы, которые начинают поворачиваться в направлении приложения поля. В этом временном интервале происходит укрупнение этих областей и возникновение областей орторомбической симметрии с локальной спонтанной поляризацией, направленной вдоль направления приложения поля. Это приводит к уменьшению оптического пропускания, что и видно на рисунке. Чем больше напряженность электрического поля (рис. 4, $a-c$ ), тем быстрее увеличиваются размеры рассеивателей. Скорость звука при этом не меняется, следовательно, никакой структурной перестройки и возникновения дальнего порядка на этом этапе не происходит. В нашем эксперименте мы использовали маленькие электрические поля, поэтому процесс возникновения новых областей орторомбической фазы и увеличение размеров рассеивателей был растянут по времени. С дальнейшим увеличением времени продолжается вращение плоскости поляризации от направления $\langle 111\rangle$ полярной оси к направлению $\langle 011\rangle$, и в итоге происходит резкий скачок в оптическом пропускании и скорости звука, связанный с изменением параметров решетки и возникновением индуцированного перехода из ромбоэдрической в орторомбическую фазу $(\mathrm{R}-\mathrm{O})$. Возникшая при комнатной температуре орторомбическая фаза оставалась стабильной в течение длительного времени и после снятия электрического поля, т.е. в достаточно низком поле $\sim 2 \mathrm{kV} / \mathrm{cm}$ в кристалле возникла необратимая орторомбическая фаза. При этом переходе одновременно меняются и оптическое пропускание, и скорость звука. Увеличение оптического пропускания в орторомбической фазе, скорее всего, связано с возникновением однодоменного состояния. Эти данные согласуются с результатами работы [19] по исследованию деформации в кристаллах PMN-32PT, в которых однодоменная орторомбическая фаза в поле [011] возникала в полях, меньших $2.2 \mathrm{kV} / \mathrm{cm}$. Как видно из рис. 4 , этот переход происходит резко, без задержек по времени. Никаких промежуточных фаз между ромбоэдрической и орторомбической фазами не возникает. Это согласуется с работами $[16,17]$ по исследованию доменной структуры в кристаллах PMN-30PT и PIN-PMN-32PT для случая приложения поля вдоль [011]. В этих работах показано, что в небольших полях до $\sim 5 \mathrm{kV} / \mathrm{cm}$ наблюдались только области с ромбической симметрией и только в больших полях $\sim 10-12 \mathrm{kV} / \mathrm{cm}$ наблюдалось два сорта доменной структуры, указывающей на сосуществование областей моноклинной и ромбической симметрии.

Интересные результаты получены для затухания звука в области фазового перехода (рис. 4, $a-c$ ). Видно, что с изменением величины приложенного поля меняется поведение затухания. В небольшом поле (рис. 4, $a$ ) наблюдается один пик затухания, связанный, по-видимому, c переориентацией областей ромбоэдрической фазы. C увеличением напряженности приложенного поля (рис. 4,c) фазовый переход проходит так быстро, что нам не удалось зафиксировать момент максимального значения акустического затухания и на рисунке видно только резкое уменьшение затухания. Наиболее сложная картина затухания наблюдается в промежуточном поле (рис. 4, $b$ ). В небольшом временном интервале видно несколько пиков затухания. Одной из вероятных причин такого поведения может быть не только переориентация областей ромбоэдрической фазы в направлении $\langle 011\rangle$, но и возникновение, и рост областей ромбической фазы 
различного размера, обуславливающих соответствующие пики затухания. Следует обратить внимание на величину затухания в ромбоэдрической и ромбической фазах. После перехода индуцируется более симметричная ромбическая фаза, поэтому затухание в ней меньше.

\section{4. Заключение}

Таким образом, в данной работе впервые проведены одновременные измерения оптических и акустических свойств в тройных системах $\mathrm{PIN}-\mathrm{PMN}-x \mathrm{PT}$ с $x=25,32 \%$.

Исследована динамика структурного упорядочения в исследованных кристаллах в различных геометриях приложения внешнего электрического поля (E $\|$ [001], $E \|[011])$ при комнатной температуре.

1. Фазовый переход в сегнетоэлектрическое состояние в исследованных кристаллах проходит в два этапа: после времени задержки, в течение которого изменение оптического пропускания и скорости звука подчиняются $\log$ зависимости, происходит резкое изменение изучаемых свойств, и кристалл переходит в фазу с дальним порядком.

2. Изменение упругих свойств наступает позже, чем изменение оптического пропускания, что связано с разными размерами полярных областей и времен релаксации, на которые реагируют свет и звук.

3. Установлено, что в интервале полей $0-2 \mathrm{kV} / \mathrm{cm}$ при $E \|[001]$ в обоих кристаллах в результате фазового перехода происходит только частичная поляризация образца.

4. В кристалле РТ32 при $E \|$ [011] в том же интервале полей $(0-2 \mathrm{kV} / \mathrm{cm})$ выявлена более сложная динамика развития сегнетоэлектрического фазового перехода из ромбоэдрической в орторомбическую фазу, завершающегося практически полной поляризацией образца.

Таким образом, совместное наблюдение изменения во времени оптических и акустических свойств позволяет проследить особенности динамики формирования полярных областей в процессе фазового перехода из стеклоподобной фазы в сегнетоэлектрическую.

\section{Список литературы}

[1] A. A. Bokov, Z.-G. Ye. J. Mater. Sci. 41, 31 (2006).

[2] G. Burns, B.A. Scott. Solid State Commun. 13, 423 (1973).

[3] V.V. Shvartsman, A.L. Kholkin. Phys. Rev. B 69, 014102 (2004).

[4] Z.-G. Ye. Curr. Opin. Solid State Mater. Sci. 6, 35 (2002).

[5] M. Iwata, K. Katsuraya, I. Suzuki, M. Maeda, N. Yasuda, Y. Ishibashi. Jpn. J. Appl. Phys. 42, 6201 (2003).

[6] S. Tsukada, S. Kojima. Phys. Rev. B 78, 144106 (2008).

[7] S. Tsukada, Y. Ike, J. Kano, T. Sekiya, Y. Shimojo, R. Wang, S. Kojima. J. Phys. Soc. Jpn. 77, 033707 (2008).

[8] B. Dkhil, P. Gemeiner, A.Al-Barakaty, L. Bellaiche, E. Dul'kin, E. Mojaev, M. Roth. Phys. Rev. B 80, 064103 (2009).

[9] E.V. Colla, M.B.Weissman. Phys. Rev. B 72, 104106 (2005).
[10] E.V. Colla, D. Vigil, J. Timmerwilke, M.B. Weissman. Phys. Rev. B 75, 214201 (2007).

[11] E.V. Colla, N. Jurik, Y. Liu, M.E.X. Delgado, M.B. Weissman, D.D. Vieland, Z.-G. Ye. J. Appl. Phys. 113, 184104 (2013).

[12] Л.С. Камзина, Л.А. Кулакова, Н. Luo. ФТТ 56, 9, 1809 (2014).

[13] Л.С. Камзина, Л.А. Кулакова. ФТТ 58, 1, 177 (2016).

[14] H. Luo, G. Xu, H. Xu, P. Wagn. Jpn. J. Appl. Phys. 39, 5581 (2000).

[15] S.J. Zhang, L. Lebrun, S. Rhee, C.A. Randall, T.R. Shrout. Appl. Phys. Lett. 81, 892 (2002).

[16] Z. Li, Z. Xu, X. Yao, Z.-Y. Cheng. J. Appl. Phys. 104, 024112 (2008).

[17] D. Lin, Z. Li, S. Zhang, Z. Xu, Xi Yao. J. Appl. Phys. 108, 034112 (2010).

[18] S. Zhang, Fei Li, Jun Luo, Ru Xia, W. Hackenberger, Thomas R. Shrout. Appl. Phys. Lett. 97, 132903 (2010).

[19] M. Shanthi, L.C. Lim. J. Appl. Phys. 106, 114116 (2009). 